\title{
ON THE BIFURCATION AND POSTBIFURCATION ANALYSIS OF ELASTIC-PLASTIC SOLIDS UNDER GENERAL PREBIFURCATION CONDITIONS
}

\author{
N. TriantafYLlidis \\ College of Engineering, University of Michigan, Ann Arbor, MI 48109, U.S.A.
}

(Received 12 July; in revised form 6 April 1983)

\begin{abstract}
IN THIS work we have studied the bifurcation and postbifurcation of elastic-plastic solids whose behavior near the critical point could not be idealized as hypoelastic and thus the "hypoelastic comparison solid" concept of R. Hill's theory is no longer applicablc. First a simplc continuous model is considered in order to illustrate the different possibilities in the stability behavior of the structures considered here. Next, a general three-dimensional stability analysis for a broad class of rate independent elastic-plastic solids is presented. It is found that for all the constitutive theories considered and for all possible prebifurcation solutions, the bifurcation functional is a simple generalization of Hill's. A completely different postbifurcation analysis is needed, however, in the case where the "hypoelastic comparison solid" concept cannot be used.
\end{abstract}

\section{INTRODUCTION}

PLASTIC buckling has been the object of intensive investigations over a long period of time. Of particular interest here is the work of HiLl $(1956,1958,1961)$ who placed the bifurcation criterion for elastic-plastic solids on a firm mathematical foundation which embraces a broad class of rate independent constitutive laws. A fundamental assumption, first introduced in a simpler context by SHANLEY (1947), in Hill's bifurcation analysis is that the plastic (or total) loading condition is satisfied everywhere in the principal solution. This requirement, which is usually met by most applications, permits the instantaneous identification at the onset of bifurcation of the elastic-plastic solid with a "hypoelastic comparison solid" in Hill's terminology. The total loading condition (sometimes called the generalized Shanley criterion) also plays an important role in the determination of the postbifurcation behavior of these structures as shown by HuTCHINSON $(1973,1974)$.

Interesting cases however exist, in which part of the prebifurcation solution of a structure is in the elastic unloading regime, as, for example, in the bifurcation under pure bending of a thick plate studied by TRIANTAFYllidis, NeEdLeman and TVERGaARD (1982) (see also, TRIANTAFYLlidis (1980)) or the puckering type of instabilities encountered in deep drawing operations in sheet metals. There a compressive buckling type of instability occurs on a part of the sheet (usually the part in contact with the punch) which is partially under elastic unloading. More recently 
TVERGAaRD (1982) investigated the buckling of axially compressed circular cylinders with axisymmetric imperfections which cause considerable deviation of the prebifurcation solution from proportional loading.

Motivated by the aforementioned examples of practical importance, an attempt was made to study the bifurcation of elastic-plastic solids under unloading and consequently to generalize the corresponding analysis in order to incorporate all possible prebifurcation histories. First, a simple continuous model is considered, similar to the one used by Hutchinson (1973), in order to illustrate the different possibilities in postbifurcation behavior of the elastic-plastic structures considered here. It is found that elastic reloading might occur in the postbifurcation regime in which case the corresponding perturbation expansion depends critically on the yield surface characteristics of the material.

Next, a general three-dimensional analysis for rate independent elastic-plastic materials is presented which incorporates not only the more classical smooth yield surface models but also the recently proposed phenomenological corner theories of CHRISTOFFERSEN and HUTCHINSON (1979). From the application standpoint, the most interesting result is that the bifurcation functional for the broad class of rate independent plasticity theories considered and for all possible prebifurcation paths, is a simple generalization of Hill's bifurcation functional. A completely different postbifurcation analysis is needed, however, in the case where the hypoelastic comparison solid concept is no longer applicable. It is shown that, for this case, a "smooth" type bifurcation occurs, i.e. not only the perturbation $\overline{\mathbf{u}}$ of the solution tends to zero as the time like parameter $\lambda$ approaches its critical value $\lambda_{\mathrm{c}}$, but the rate $\dot{\overline{\mathbf{u}}} \rightarrow 0$ for $\lambda \rightarrow \lambda_{\mathrm{c}}$ as well.

\section{Simple Model}

In order to understand better the bifurcation and postbifurcation behavior of elastic-plastic solids whose constitutive response cannot be idealized as hypoelastic in the vicinity of the bifurcation point, we make use of a simple continuous model, much in the spirit of the rigid T models employed by HUTCHINSON (1974).

Our model structure consists of a rigid rectangular plate $\mathrm{AA}^{\prime} \mathrm{B}^{\prime} \mathrm{B}$ of dimensions $\mathrm{AB}$ $=2 a, \mathrm{AA}^{\prime}=2 b$. A vertical rod $\mathrm{OD}$ of length $L$ is rigidly attached to its center $\mathrm{O}$ as shown in Fig. 1. The edges $A B$ and $A^{\prime} B^{\prime}$ of the plate rest on a continuous line of elasticplastic springs with the following force $(\sigma)$-displacement $(\varepsilon)$ response

$$
\dot{\sigma}=\hat{L} \dot{\varepsilon} ; \quad \hat{L}= \begin{cases}E & \text { if } \varepsilon>0 \quad \text { and } \varepsilon<\varepsilon_{\max }, \\ {\left[E+\left(E_{\mathrm{t}}-E\right) H(\hat{\varepsilon})\right]} & \text { otherwise, } \quad\left(E>E_{\mathrm{q}}>0\right),\end{cases}
$$

where $\varepsilon_{\max }$ denotes the maximum strain attained during the most recent loading cycle. Here and subsequently () denotes differentiation with respect to some monotonically increasing parameter $\lambda$ (also called a "time like" parameter) and $H(x)$ is the Heaviside step function $(H(x)=0$ for $x<0, H(x)=1$ for $x \geqslant 0)$. From the kinematics of the model it can be easily deduced that

$$
\varepsilon=u+x \theta+y \psi
$$

with $u$ the vertical displacement of the plate's center $O$ and $\psi, \theta$ the (small) rotations of OD about the $x$ and $y$ axes. 


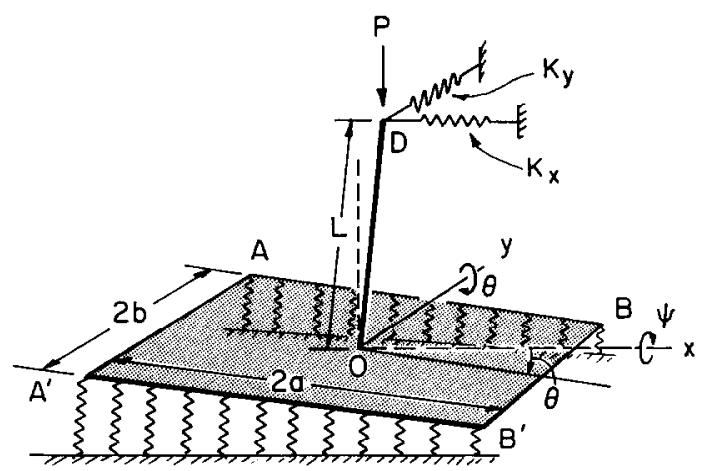

FIG. 1. Continuous model of a rigid rectangular plate resting on two lines of inelastic springs.

A vertical force $P$ is applied to the end D of the rod OD. In order to introduce into the model the destabilizing effects of geometric nonlinearities that are usually present in more complicated (and more realistic) structures, we have attached at point D two springs with secant stiffnesses $K_{x}=s \psi / \theta L$ and $K_{y}=(K+A \theta) / L$ along the $x$ and $y$ directions, as shown in Fig. 1.

The angle $\theta$ has been chosen to play the role of the monotonically increasing "time like" parameter $\lambda$. Here it will be assumed that $\theta>0$ and the issue of bifurcation with respect to $\psi$ will be examined (the trivial solution to the equilibrium equations for the rigid plate has $\psi \equiv 0$ ). It is not difficult to show that the geometric and material propertics of the structure can be chosen in such a way that a bifurcation with respect to $\theta$ always precedes any bifurcation with respect to $\psi$, i.e. $P_{\theta}^{\mathrm{c}}<P_{\psi}^{\mathrm{c}}$. Two cases will be distinguished here.

Case 1. $s \neq 0$ (asymmetric structure)

Assuming no elastic reloading in the structure (the validity of this hypothesis will be verified a posteriori), the incremental force equilibrium with respect to the vertical direction gives

$$
2\left(E+E_{\mathrm{t}}\right) a \dot{u}+\left(E_{\mathrm{t}}-E\right)\left(a^{2}+\dot{u}^{2}+\dot{\psi}^{2} b^{2}\right)=\dot{P} .
$$

Similarly, the incremental moment equilibrium with respect to the $y$ axis yields

$$
\left(E+E_{\mathrm{t}}\right) \frac{2 a^{3}}{3}+\left(E-E_{\mathrm{t}}\right)\left(-a^{2}+\dot{\psi}^{2} b^{2}+\frac{\dot{u}^{2}}{3}\right) \dot{u}=(P \theta+s \psi) \dot{L}
$$

and the corresponding moment equilibrium equation with respect to the $x$ axis is

$$
\left(E+E_{\mathrm{t}}\right) 2 a b^{2} \dot{\psi}+\left(E_{\mathrm{t}}-E\right) 2 b^{2} \dot{u} \dot{\psi}=[(P+K+A \theta) \psi]^{\circ} L .
$$

The following perturbation expansions for $u, P, \psi$ are adopted:

$$
\begin{aligned}
P & =P_{0}+\bar{P}=P_{0}(\theta)+P_{2} \xi^{2}+P_{3} \xi^{3}+\ldots \\
u & =u_{0}+\bar{u}=u_{0}(\theta)+u_{2} \xi^{2}+u_{3} \xi^{3}+\ldots \\
\psi & =0+\bar{\psi}=0+\psi_{2} \xi^{2}+\psi_{3} \xi^{3}+\ldots ; \quad \xi \equiv \theta-\theta_{\mathrm{c}}
\end{aligned}
$$


where, here and subsequently, a quantity surmounted by a bar $(0$ will designate the change of values of the quantity in question between the principal and bifurcated paths, while a superscript or subscript zero indicates evaluation on the principal branch of the solution. Moreover, a superscript or subscript (c) is used when a quantity is computed at the onset of bifurcation.

Substitution of (2.6) in the incremental equilibrium equations (2.3)-(2.5) and expansion about the critical angle $\theta_{\mathrm{c}}$ up to order $\xi$ yields the following three equations :

$$
\begin{gathered}
{\left[2 a\left(E+E_{\mathrm{t}}\right)+2 \dot{u}_{\mathrm{c}}\left(E_{\mathrm{t}}-E\right)\right] u_{2}=P_{2},} \\
{\left[\left(E_{\mathrm{t}}-E\right)\left(a^{2}-\dot{u}_{\mathrm{c}}^{2}\right)\right] u_{2}-\theta_{\mathrm{c}} L P_{2}=s L \psi_{2},} \\
b^{2}\left[2 a\left(E+E_{\mathrm{t}}\right)+2 \dot{u}_{\mathrm{c}}\left(E_{\mathrm{t}}-E\right)\right]=\left(P_{\mathrm{c}}+K+A \theta_{\mathrm{c}}\right) L,
\end{gathered}
$$

while the $\xi^{2}$ term of (2.5) in combination with (2.9) gives

$$
2\left(E_{\mathrm{t}}-E\right) b^{2}\left[\ddot{u}_{\mathrm{c}}+2 u_{2}\right]=\frac{3}{2}\left(P_{\mathrm{c}}+A\right) L .
$$

The bifurcation condition providing the critical angle $\theta_{\mathrm{c}}$ is given by (2.9) while from (2.7), (2.8) and (2.10) the amplitude of the bifurcation mode $\psi_{2}$ is found to be

$$
\begin{aligned}
\psi_{2}=\left(-\ddot{u}_{\mathrm{c}}\right) \frac{E-E_{\mathrm{t}}}{2 s L}\left[\frac{3\left(\dot{P}_{\mathrm{c}}+A\right) L}{4 b^{2}\left(E-E_{\mathrm{t}}\right)\left(-\ddot{u}_{\mathrm{c}}\right)}-1\right] \\
\times\left[a^{2}-\dot{u}_{\mathrm{c}}^{2}+2 \theta_{\mathrm{c}} L\left(\frac{E+E_{\mathrm{t}}}{E-E_{\mathrm{t}}} a-\dot{u}_{\mathrm{c}}\right)\right] \equiv \frac{\hat{\psi}_{2}}{2 s b}\left(-\ddot{u}_{\mathrm{c}}\right) .
\end{aligned}
$$

The validity of the no elastic reloading assumption must now be verified. For $s>0$ the no elastic reloading condition is ensured if $\dot{\varepsilon}=\dot{\varepsilon}_{0}+\dot{\bar{\varepsilon}}<0$ in the unloading zone with $y=+b$, i.e. when

$$
\dot{u}_{\mathrm{c}}+\left(2 u_{2}+\ddot{u}_{\mathrm{c}}\right) \xi+x+2 \psi_{2} b \xi+0\left(\xi^{2}\right)<0, \quad\left(-a \leqslant x \leqslant-\dot{u}_{\mathrm{c}}\right) .
$$

Since the unloading zone in the prebifurcation solution is $-a \leqslant x \leqslant-\dot{u}_{\mathrm{c}}$ at $\theta=\theta_{\mathrm{c}}$, no elastic reloading will be encountered if the neutral loading point $x=-\dot{u}_{\mathrm{c}}$ continues to unload after bifurcation and from (2.12) one deduces the condition

$$
2 u_{2}+\ddot{u}_{\mathrm{c}}+2 \psi_{2} b<0
$$

which, with the help of (2.7), (2.8), (2.10) and (2.11), can be equivalently rewritten as

$$
s>s_{\min }=\hat{\psi}_{2} /\left[\frac{3\left(\dot{P}_{\mathrm{c}}+A\right) L}{4\left(E-E_{\mathrm{t}}\right)\left(-\ddot{u}_{\mathrm{c}}\right) b^{2}}\right] .
$$

Using (2.11) it can be easily verified that $s_{\min }>0$ since $\ddot{u}_{0}<0, a>\left|\dot{u}_{0}\right|$ (in view of the propagation of the unloading zone in the $+x$ direction) and

$$
\left(\dot{P}_{\mathrm{c}}+A\right) L>2\left(E-E_{\mathrm{t}}\right)\left(-\ddot{u}_{\mathrm{c}}\right) b^{2}
$$

(which follows from (2.9)). For $0<s<s_{\min }$ elastic reloading will be present in the postbifurcation solution and thus a reformulation of the governing equations $(2.3)-(2.5)$ is needed. 


\section{Case 2. $s=0$ (symmetric structure)}

As we have seen in the previous case, elastic reloading will be present in the postbifurcation solution for any $s<s_{\min }$. Without loss of generality we can assume $\psi$, $\dot{\psi}>0$ near $\theta_{\mathrm{c}}$ which in turn implies the presence of elastic reloading on the side $\mathrm{AB}$ $(y=+b)$ of the plate (see Fig. 1). Let $x_{n}(\theta)$ denote the $x$ coordinate of the neutral loading point in $\mathrm{AB}$ and $x_{r}(\theta)$ denote the corresponding coordinate of the point separating the elastic $\left(x<x_{r}\right)$ from the plastic $\left(x>x_{r}\right)$ reloading zone. The three incremental equilibrium equations in this case assume the form

$$
\begin{gathered}
2\left(E+E_{\mathrm{t}}\right) a \dot{u}+\left(E_{\mathrm{t}}-E\right)\left(a^{2}+\dot{u}^{2}+\dot{\psi}^{2} b^{2}\right)+\frac{E-E_{\mathrm{t}}}{2}\left(x_{r}-x_{n}\right)^{2}=\dot{P} \\
\left(E+E_{\mathrm{t}}\right) \frac{2}{3} a^{3}+\left(E-E_{\mathrm{t}}\right)\left(-a^{2}+\dot{\psi}^{2} b^{2}+\frac{\dot{u}^{2}}{3}\right) \dot{u}+\frac{E-E_{\mathrm{t}}}{6}\left(x_{r}-x_{n}\right)^{2}\left(2 x_{r}+x_{n}\right)=(P \theta) L \\
\left(E+E_{\mathrm{t}}\right) 2 a b \dot{\psi}+\left(E_{\mathrm{t}}-E\right) 2 b^{2} \dot{u} \dot{\psi}+\frac{E-E_{\mathrm{t}}}{2} b\left(x_{r}-x_{n}\right)^{2}=[(P+K+A \theta) \psi]^{\circ} L .
\end{gathered}
$$

Notice that the above equations are similar to (2.3)-(2.5) with the addition of the $\left(x_{r}-x_{n}\right)^{2}$ term due to the presence of elastic reloading. The perturbation expansions for $P, u, \psi$ are still given by (2.6) (the only difference in this case being that $P_{2}=u_{2}=0$, as can be easily verified) and consequently the corresponding expansions for $x_{n}$ and $x_{r}$ will assume the form

$$
\begin{aligned}
& x_{n}=x_{n}^{0}+\bar{x}_{n}=x_{n}^{0}(\theta)+x_{n}^{1} \xi+\ldots, \\
& x_{r}=x_{r}^{0}+\bar{x}_{r}=x_{n}^{0}(\theta)+x_{r}^{1} \xi+\ldots
\end{aligned}
$$

In the above expressions, $x_{r}^{0}(\theta)=x_{n}^{0}(\theta)$, in view of the absence of the clastic reloading zone in the prebifurcation solution. The coefficient $x_{n}^{1}$ in the expansion for $\bar{x}_{n}$ is found from the definition of $x_{m}$, i.e. $\dot{\varepsilon}\left(x_{n}\right)=0$, in conjunction with (2.18), to be

$$
x_{n}^{1}=-2 \psi_{2} b \text {. }
$$

The determination of $x_{r}^{1}$ in terms of $\psi_{2}$ is a somewhat more complicated matter that proceeds as follows. For a given point with coordinates $(x, b)$, the maximum displacement $\varepsilon_{\mathrm{m}}(x)$ achieved in the prebifurcation solution occurs when $\theta=\theta_{\mathrm{m}}(x)$, with $\theta_{\mathrm{m}}(x)$ satisfying

$$
\dot{u}_{0}\left(\theta_{\mathrm{m}}\right)+x=0, \quad \theta_{\mathrm{m}}(x)=\left(\dot{u}_{0}\right)^{-1}(-x) .
$$

The corresponding maximum displacement $\varepsilon_{\max }(x)$ can be written as

$$
\varepsilon_{\mathrm{m}}(x)=u_{0}\left[\theta_{\mathrm{m}}(x)\right]+x \theta_{\mathrm{m}}(x) .
$$

Following the onset of a bifurcation $(\psi>0)$ the coordinate $x_{r}$ of the point separating the elastic from the plastic reloading regions can be calculated by solving the equation $\varepsilon\left(x_{r}\right)=\varepsilon_{\mathrm{m}}\left(x_{r}\right)$ which, with the help of $(2.21),(2.18)$, takes the form

$$
u_{0}+\bar{u}+\theta\left(x_{n}^{0}+\bar{x}_{r}\right)+\bar{\psi} b=u_{0}\left[\theta_{m}\left(x_{n}^{0}+\bar{x}_{r}\right)\right]+\left(x_{n}^{0}+\bar{x}_{r}\right) \theta_{m}\left(x_{n}^{0}+\bar{x}_{r}\right) .
$$

Expanding (2.22) about the critical angle $\theta_{c}$ and using (2.18)-(2.20), one obtains, by 
collecting all the $O\left(\xi^{2}\right)$ terms, the following expression for $x_{r}^{1}$ :

$$
x_{r}^{1}=-\sqrt{2 \psi_{2} b\left(-\ddot{u}_{c}\right)} .
$$

Using (2.23) together with (2.19) and (2.18) in the equilibrium equation (2.17), we obtain from the $O(\xi)$ terms the bifurcation condition

$$
2 b^{2}\left[\left(E+E_{\mathrm{t}}\right) a+\left(E_{\mathrm{t}}-E\right) \dot{u}_{\mathrm{c}}\right]=\left[P_{\mathrm{c}}+K+A \theta_{\mathrm{c}}\right] L,
$$

while from the $O\left(\xi^{2}\right)$ terms the bifurcation mode amplitude $\psi_{2}$ is found to be

$$
\psi_{2}=\frac{\left(-\ddot{u}_{\mathrm{c}}\right)}{2 b}\left\{1+\left[\frac{3\left(\dot{P}_{\mathrm{c}}+A\right) L}{\left(E E_{\mathrm{t}}\right) b^{2}\left(\cdots \ddot{u}_{\mathrm{c}}\right)}-4\right]^{1 / 2}\right\}^{2} .
$$

Note that the bifurcation condition (2.24) coincides with (2.9), as expected. Making use of (2.24) and the assumption introduced in the heginning of this section that a bifurcation with respect to $\theta$ precedes any $\psi$ bifurcation, i.e. $P_{\theta}^{\mathrm{c}}<P_{\psi}^{\mathrm{c}} \equiv P_{\mathrm{c}}, K$ and $A$ can be always chosen so as to ensure the reality of $\psi_{2}$ in (2.25). It is understood that the same analysis could have been repeated for $\psi<0$ providing the symmetric bifurcation branch (Fig. 2).

Two interesting conclusions can be drawn from the study of the simple model analysed here, for structures that violate the plastic loading condition in the prebifurcation state.

First, the dependence of the eigenmode amplitude $\psi$ on the time like parameter $\theta$ is of the type $\psi=\psi_{2}\left(\theta-\theta_{c}\right)^{2}+O\left(\theta-\theta_{c}\right)^{3}$ which shows that, for this type of problem, not only the displacements $\mathbf{u}(\mathbf{u}=(u, \theta, \psi))$ are continuous near the critical load $\theta_{\mathrm{c}}$ but the rates $\mathrm{du} / \mathrm{d} \theta$ are also continuous at the same point. Notice the difference with the case of plastic bifurcation from a total loading prebifurcation solution, where $\mathbf{u} \rightarrow \mathbf{u}_{\mathrm{c}}$ as $\theta \rightarrow \theta_{\mathbf{c}}$ but $\mathrm{d} \mathbf{u} / \mathrm{d} \theta \rightarrow \mathrm{d} \mathbf{u}_{\mathrm{c}} / \mathrm{d} \theta$ as $\theta \rightarrow \theta_{\mathrm{c}}$.

The second conclusion is that elastic reloading is not necessarily present in the postbifurcation solution (as seen in the case of the asymmetric structure with $s>s_{\min }$ ). When elastic reloading is present in the postbifurcation state, the asymptotic analysis
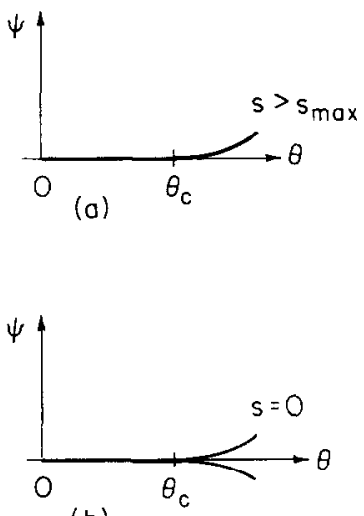

(b)

Fig. 2. Typical bifurcation for the asymmetric (a) and symmetric (b) plate models. 
has to take into account the shape of the yield surface $\left(\varepsilon_{\max }\right.$ in the case of the onedimensional model treated here). The results obtained thus far via the simplified plate model will now be properly generalized for a three-dimensional elastic-plastic solid.

\section{Generalizations to Three-Dimensional Continua}

This section deals with the bifurcation and postbifurcation analysis of a wide class of elastic-plastic solids whose constitutive response cannot, in general, be idealized as hypoelastic in the vicinity of the bifurcation load. The basic ideas for the subsequent analysis are drawn from the analysis of the simple model presented in the previous section.

Consider a body occupying a volume $V$ and bounded by a smooth surface $\partial V$ in some reference configuration which will be kept fixed at all stages of the motion (full Lagrangian description of the motion). To avoid indices, frame invariant dyadic notation will be used for the rest of this section. In the interest of simplicity the first Piola-Kirchhoff tensor $T$ will be used as a stress measure and the deformation gradient $\mathbf{F} \equiv \mathbf{x} \boldsymbol{\nabla}$ (where $\mathbf{x}$ denotes the current position vector of a given material point, and $\boldsymbol{\nabla}$ is the gradient operator with respect to the reference configuration) will play the role of a deformation measure. A quantity surmounted by a () denotes the derivative with respect to some monotonically increasing parameter $\lambda$ (also termed "time like"). Most of the rate independent theories of plasticity can be put in the form

$$
\dot{\mathbf{T}}^{\mathrm{T}}=\mathbf{L}\left(\mathbf{T}, \mathbf{F}, \dot{\mathbf{F}}_{i} q\right): \dot{\mathbf{F}}, \dagger
$$

where the fourth rank tensor of incremental moduli $L$ in general depends on the current stress and deformation state, the deformation rate + as well as on a set of scalar parameters ${ }_{i} q(i=1, \ldots, N)$, termed internal variables, that represent the past history. Their current values can be determined via evolution equations of the type

$$
{ }_{i} \dot{q}=Q_{i}\left(\mathbf{T}, \mathbf{F}, \mathbf{i}, \dot{\mathbf{F}},{ }_{j} q\right) \text {. }
$$

Usually, in most of the rate independent plasticity theories some additional structure in the constitutive equations is available in view of the existence of a rate potential $W$ (homogeneous of degree 2 in $\dot{\mathbf{F}}$ ) with the property

$$
\mathbf{L}=\frac{\partial^{2} W\left(\mathbf{T}, \mathbf{F}, \dot{\mathbf{F}},{ }_{j} q\right)}{\partial \dot{\mathbf{F}} \partial \dot{\mathbf{F}}} .
$$

The symmetrics in $\mathrm{L}$ implicd by (3.3) will prove very helpful in the subsequent use of the principle of virtual work for the general bifurcation analysis. Cases exist, as for example in the corner theories of CHRISTOFFERSEN and HUTCHINSON (1979) where $W$ is given as a function of $T$ instead of $F$ and consequently the constitutive equation corresponding to (3.1) appears in the form $\mathbf{F}=\mathbf{M}\left(\mathbf{T}, \mathbf{F}, \dot{\mathbf{T}},{ }_{i q} q\right): \dot{\mathbf{T}}^{\mathrm{T}}$. In principle the relation can be inverted and put in the form of (3.1) but an explicit analytical expression is not possible except in

† In Cartesian coordinates: $\dot{T}_{i j}^{\mathrm{T}}=L_{i j k l} \dot{F}_{k l}$.

$\ddagger$ For rate independent theories of plasticity the $\mathbf{L}$ dependence on $\mathbf{F}$ is homogeneous of degree zero, i.e. $\mathbf{L}$ depends on $\dot{\mathbf{F}} /\|\dot{\mathbf{F}}\|$. 
some special cases, as explained in Christoffersen and HutChinson (1979). Consequently, and without loss of generality, the constitutive equations (3.1) will be used in view of the resulting simplifications in the algebraic manipulations. Additionally, it should be mentioned that the dependence of $\mathbf{L}$ and $Q_{i}$ on their arguments is assumed to be sufficiently smooth to ensure a meaning for the operations needed by the subsequent analysis.

Departing from the principle of incremental virtual work

$$
\int_{V} \delta \mathbf{F}: \mathbf{T}^{\mathrm{T}}=\int_{V} \mathbf{f} \cdot \delta \mathbf{x}+\int_{\partial V} \dot{\mathbf{t}} \cdot \delta \mathbf{x},
$$

where $f$ is the body force (per unit reference volume) and the pseudotraction (per unit reference area), $\dagger$ and expanding (3.4) about the principal branch of the solution, one obtains, with the help of (3.1) and (3.2),

$$
\begin{aligned}
& \int_{V} \delta \mathbf{F}:\left\{\mathbf{L}_{0}+\left.\frac{\partial \mathbf{L}}{\partial \mathbf{T}}\right|_{0}: \overline{\mathbf{T}}+\left.\frac{\partial \mathbf{L}}{\partial \mathbf{F}}\right|_{0}: \overline{\mathbf{F}}+\left.\frac{\partial \mathbf{L}}{\partial \dot{\mathbf{F}}}\right|_{0}: \dot{\overline{\mathbf{F}}}+\left.\sum_{i} \frac{\partial \mathbf{L}}{\partial_{i} q}\right|_{0}{ }_{i} \bar{q}+\frac{1}{2 !}\left[\left(\left.\frac{\partial^{2} \mathbf{L}}{\partial \dot{\mathbf{F}} \partial \dot{\mathbf{F}}}\right|_{0}: \dot{\overline{\mathbf{F}}}\right): \dot{\overline{\mathbf{F}}}+\ldots\right]\right. \\
& \left.+\frac{1}{3 !}\left[\left(\left(\left.\frac{\partial^{3} \mathbf{L}}{\partial \dot{\mathbf{F}} \partial \dot{\mathbf{F}} \partial \dot{\mathbf{F}}}\right|_{0}: \dot{\overline{\mathbf{F}}}\right): \dot{\mathbf{F}}\right): \dot{\overline{\mathbf{F}}}+\ldots\right]+\ldots\right\}:\left\{\dot{\mathbf{F}}_{0}+\dot{\overline{\mathbf{F}}}\right\}=\int_{V} \mathbf{f}_{0} \cdot \partial \mathbf{x}+\int_{\partial V} \dot{\mathbf{t}}_{0} \cdot \partial \mathbf{x}, \dot{t}
\end{aligned}
$$

with the perturbations $\overline{\mathbf{F}}, \overline{\mathbf{T}}$ and $i \bar{q}$ satisfying

$$
\begin{aligned}
\mathbf{F}_{0}+\overline{\mathbf{F}}=\mathbf{x}_{0} \boldsymbol{\nabla}+\overline{\mathbf{u}} \boldsymbol{\nabla} ; \quad \dot{\overline{\mathbf{T}}}_{0}^{\mathrm{T}}+\dot{\overline{\mathbf{T}}}^{\mathrm{T}}=\left[\mathbf{L}_{0}+\left.\frac{\partial \mathbf{L}}{\partial \dot{\mathbf{F}}}\right|_{0}: \dot{\overline{\mathbf{F}}}+\ldots\right]:\left[\dot{\mathbf{F}}_{0}+\dot{\overline{\mathbf{F}}}\right] \\
{ }_{i} \dot{q}^{0}+{ }_{i} \dot{\bar{q}}=Q_{i}^{0}+\left.\frac{\partial Q_{i}}{\partial \dot{\mathbf{T}}}\right|_{0}: \dot{\mathbf{T}}+\left.\frac{\partial Q_{i}}{\partial \dot{\mathbf{F}}}\right|_{0}: \dot{\overline{\mathbf{F}}}+\ldots
\end{aligned}
$$

In analogy with (2.6), the following postbifurcation expansions for $\overline{\mathbf{F}}, \overline{\mathbf{T}}, \bar{q}$ will be used.

$$
\begin{array}{ll}
\overline{\mathbf{u}}=\mathbf{u}_{\alpha_{1}} \xi^{\alpha_{1}}+\mathbf{u}_{\alpha}, \xi^{\alpha_{2}}+\ldots, & \overline{\mathbf{F}}=\mathbf{F}_{\alpha_{1}} \xi^{\alpha_{1}}+\mathbf{F}_{\alpha_{2}} \xi^{\alpha_{2}}+\ldots, \\
\overline{\mathbf{T}}=\mathbf{T}_{\alpha_{1}} \xi^{\alpha_{1}}+\mathbf{T}_{\alpha_{2}} \xi^{\alpha_{2}}+\ldots, & { }_{i} \bar{q}={ }_{i} q_{\alpha_{1}} \xi^{\alpha_{1}}+{ }_{i} q_{\alpha_{2}} \xi^{\alpha_{2}}+\ldots,
\end{array}
$$

where $\xi \equiv \lambda-\lambda_{e}$ and $1<\alpha_{1}<\alpha_{2}<\ldots$ Introducing (3.7) into (3.5), (3.6) and expanding about the critical value $\lambda_{\mathrm{c}}$ of the time like parameter, one obtains (using also (3.3)) for the lowest order term $O\left(\xi^{\alpha_{1}-1}\right)$,

$$
\int_{V} \delta \mathbf{F}:\left[\mathbf{L}_{\mathrm{c}}+\left.\frac{\partial \mathbf{L}}{\partial \dot{\mathbf{F}}}\right|_{\mathrm{c}}: \dot{\mathbf{F}}_{\mathrm{c}}\right]: \mathbf{F}_{\alpha_{1}}=0, \S
$$

or, equivalently, in view of the homogeneous dependence of $\mathbf{L}$ on $\mathbf{F}$ (of degree zero),

$$
\int_{V} \delta \mathbf{F}: \mathbf{L}_{\mathfrak{c}}: \mathbf{F}_{\alpha_{1}}=0 .
$$

All external loads acting on the body are assumed to be dead loads.

+ The Cartesian coordinates of the six rank tensor $\partial \mathbf{L} / \partial \mathbf{F}$ are $\partial L_{i j k l} / \partial F_{m n}$, those of the eight rank tensor $\partial^{2} \mathbf{L} / \partial \mathbf{F} \partial \mathbf{F}$ are $\partial L_{i j k} / \partial F_{m n} \partial F_{r s}$ and analogous expressions hold for the other derivatives.

SThe existence of some (additional) essential boundary conditions for $\mathbf{y}_{x_{x}}$ is tacitly assumed. 
The above equation provides the critical value of $\lambda$ at which bifurcation is possible as well as the corresponding eigenmode $\mathbf{u}_{\alpha_{1}}\left(\mathbf{F}_{\alpha_{1}}=\mathbf{u}_{\alpha_{1}} \nabla\right)$. It can be shown that positive definiteness of the self adjoint linear operator defined by the left hand side of (3.8) excludes bifurcation from the reference state $(\lambda=0)$ up until the lowest eigenvalue $\lambda_{c}$ of (3.8).

In order to specify the amplitude $A_{\alpha_{1}}$ of the eigenmode $\mathbf{u}_{\alpha_{1}}$ as well as the exponents $\alpha_{1}, \alpha_{2}, \ldots$, one takes the terms of next higher order in $\xi$ in the expansion of (3.5) about $\lambda_{c}$, namely

$$
\begin{aligned}
& \int_{V} \delta \mathbf{F}:\left\{\left[\left(\left.\frac{\partial \mathbf{L}}{\partial \mathbf{T}}\right|_{\mathrm{c}}: \mathbf{T}_{\alpha_{1}}+\left.\frac{\partial \mathbf{L}}{\partial \mathbf{F}}\right|_{\mathbf{c}}: \mathbf{F}_{\alpha_{1}}+\left.\sum_{i} \frac{\partial \mathbf{L}}{\partial_{i} q}\right|_{\mathrm{c}}{ }_{i} q_{\alpha_{1}}\right): \dot{\mathbf{F}}_{\mathrm{c}}\right.\right. \\
& \left.+\alpha_{1}\left(\left(\frac{\partial \mathbf{L}}{\partial \dot{\mathbf{F}}}\right)_{\mathrm{c}}: \dot{\mathbf{F}}_{\mathrm{c}}+\left.\frac{\partial \mathbf{L}}{\partial \dot{\mathbf{F}}}\right|_{\mathrm{c}}: \ddot{\mathbf{F}}_{\mathrm{c}}+\dot{\mathbf{L}}_{\mathrm{c}}\right): \mathbf{F}_{\alpha_{1}}\right] \xi^{\alpha_{1}} \\
& +\left(\alpha_{1}\right)^{2}\left[\left(\left(\left.\frac{1}{2 !} \frac{\partial^{2} \mathbf{L}}{\partial \dot{\mathbf{F}} \partial \dot{\mathbf{F}}}\right|_{\mathbf{c}}: \dot{\mathbf{F}}_{\mathbf{c}}+\left.\frac{\partial \mathbf{L}}{\partial \dot{\mathbf{F}}}\right|_{\mathrm{c}}\right): \mathbf{F}_{\alpha_{1}}\right): \mathbf{F}_{\alpha_{1}}\right] \xi^{2\left(\alpha_{1}-1\right)} \\
& +\left(\alpha_{1}\right)^{3}\left[\left(\left(\left(\left.\frac{1}{3 !} \frac{\partial^{3} \mathbf{L}}{\partial \dot{\mathbf{F}} \partial \dot{\mathbf{F}} \partial \dot{\mathbf{F}}}\right|_{\mathrm{c}}: \dot{\mathbf{F}}_{\mathrm{c}}+\frac{1}{2 !} \frac{\partial^{2} \mathbf{L}}{\partial \dot{\mathbf{F}} \partial \dot{\mathbf{F}}}\right): \mathbf{F}_{\alpha_{1}}\right): \mathbf{F}_{\alpha_{1}}\right): \mathbf{F}_{\alpha_{1}}\right] \xi^{3\left(\alpha_{1}-1\right)} \\
& \left.+\alpha_{2}\left[\left(\mathbf{L}_{\mathrm{c}}+\left.\frac{\partial \mathbf{L}}{\partial \dot{\mathbf{F}}}\right|_{\mathrm{c}}: \dot{\mathbf{F}}_{\mathrm{c}}\right): \mathbf{F}_{\alpha_{2}}\right] \xi^{\alpha_{2}-1}+\ldots\right\}=0
\end{aligned}
$$

with $\mathbf{T}_{\alpha_{1}},{ }_{i} q_{\alpha_{1}}$ given in terms of $\mathbf{F}_{\alpha_{1}}$ by

$$
\mathbf{T}_{\alpha_{1}}^{\mathrm{T}}=\mathbf{L}_{\mathrm{c}}: \mathbf{F}_{\alpha_{1}}, \quad{ }_{i} q_{\alpha_{1}}=\left.\frac{\partial Q_{i}}{\partial \dot{\mathbf{F}}}\right|_{\mathrm{c}}: \mathbf{F}_{\alpha_{1}}+\left.\frac{\partial Q_{i}}{\partial \dot{\mathbf{T}}}\right|_{\mathrm{c}}: \mathbf{T}_{\alpha_{1}} ; \quad\left(\mathbf{F}_{\alpha_{1}}=\mathbf{u}_{\alpha_{1}} \nabla\right)
$$

By putting $\delta \mathbf{F}=\mathbf{F}_{\alpha_{1}}$, the term in $\xi^{\alpha_{2}-1}$ vanishes in view of (3.8). Assuming that the resulting coefficient of $\xi^{2\left(\alpha_{1}-1\right)}$ is non zero, the perturbation analysis goes through only if $\alpha_{1}=2\left(\alpha_{1}-1\right)=2$. From (3.9), (3.10) and after making use of the homogeneous dependence of $\mathbf{L}$ on $\mathbf{F}$ (i.e. $(\partial \mathbf{L} / \partial \mathbf{F}): \mathbf{F}=0)$, the amplitude $A_{2}$ of the eigenmode $\mathbf{u}_{2}$ is found to be

$$
A_{2}=-\frac{\int_{V} \hat{\mathbf{F}}_{2}:\left[\frac{1}{2}\left(\left.\frac{\partial \mathbf{L}}{\partial \mathbf{T}}\right|_{\mathrm{c}}: \hat{\mathbf{T}}_{2}+\left.\frac{\partial \mathbf{L}}{\partial \mathbf{F}}\right|_{\mathrm{c}}: \hat{\mathbf{F}}_{2}+\left.\sum_{i} \frac{\partial \mathbf{L}}{\partial_{i} q}\right|_{\mathrm{c}} \hat{q}_{2}\right): \dot{\mathbf{F}}_{\mathrm{c}}+\dot{\mathbf{L}}_{\mathrm{c}}\right]: \hat{\mathbf{F}}_{2}}{\int_{V} \hat{\mathbf{F}}_{2}:\left(\left.\frac{\partial \mathbf{L}}{\partial \dot{\mathbf{F}}}\right|_{\mathrm{c}}: \hat{\mathbf{F}}_{2}\right): \hat{\mathbf{F}}_{2}}
$$

where $\hat{\mathbf{u}}_{2}$ is the eigenmode, normalized in some convenient fashion $\left(\hat{\mathbf{F}}_{2}, \hat{\mathbf{T}}_{2}\right.$, ${ }_{i} \hat{q}_{2}$ correspond to $\hat{\mathbf{u}}_{2}$ ). The postbifurcation analysis can be carried out to the higher order terms in the following fashion. Assuming that the eigenmode $\mathbf{u}_{2}$ is unique, put $\mathbf{F}_{i}=\hat{\mathbf{F}}_{i}+A_{i} \hat{\mathbf{F}}_{2}(i>2)$ where $\hat{\mathbf{F}}_{i}$ is orthogonal (in an appropriate sense) to $\hat{\mathbf{F}}_{2}$. To compute $A_{i}$, put $\delta \mathbf{F}=\hat{\mathbf{F}}_{2}$ in (3.9) and take the term of order $\xi^{i}$ into account. Thus, the postbifurcation expansion for $\overline{\mathbf{u}}$ can (in principle) be written as

$$
\overline{\mathbf{u}}=A_{2} \hat{\mathbf{u}}_{2} \xi^{2}+\left(A_{3} \hat{\mathbf{u}}_{2}+\hat{\mathbf{u}}_{3}\right) \xi^{3}+\left(A_{4} \hat{\mathbf{u}}_{2}+\hat{\mathbf{u}}_{4}\right) \xi^{4}+\ldots
$$

In the case where the denominator of (3.11) vanishes, the next possible choice in (3.9) for 
$\alpha_{1}$ is $\alpha_{1}=3\left(\alpha_{1}-1\right)=1.5$ and the corresponding expansion for $\overline{\mathbf{u}}$ will take the form :

$$
\overline{\mathbf{u}}=A_{1.5} \hat{\mathbf{u}}_{1.5} \xi^{1.5}+\left(A_{2} \hat{\mathbf{u}}_{1.5}+\hat{\mathbf{u}}_{2}\right) \xi^{2}+\left(A_{2.5} \hat{\mathbf{u}}_{1.5}+\hat{\mathbf{u}}_{2.5}\right) \xi^{2.5}+\ldots
$$

In the last case we should note that, since the equation for $A_{1.5}$ is quadratic, we have the possibility of nonreal bifurcation branches.

The first important implication of the preceding analysis is, following from (3.8), that for all independent elastic-plastic materials (i.e. materials obeying a constitutive equation of the type (3.1)), irrespective of the presence or not of a corner on the yield surface and for arbitrary prebifurcation solutions, the bifurcation functional is still given by

$$
F(\lambda, \mathbf{v})=\int_{V}(\mathbf{v} \nabla): \mathbf{L}_{0}:(\mathbf{v} \mathbf{V}) \mathrm{d} V, \dagger
$$

where the incremental moduli $\mathbf{L}_{0}$ are now evaluated on the principal branch of the solution (Hill's hypoelastic comparison moduli coincide with $\mathbf{L}_{0}$ for total loading in the principal solution). Similar arguments to those presented by HILL $(1956,1958)$ show that bifurcation becomes possible for the first time (as $\lambda$ increases from zero) when $F$ loses its positive definiteness.

The next important conclusion, which can be drawn from the form of the postbifurcation expansions (3.12), (3.13), is that in the general case when the material cannot be idealized as a hypoelastic one at the onset of bifurcation, the bifurcation point is a cusp point (see Fig. 2); this means that, in a generalized load-generalized displacement diagram, the bifurcated branch of the solution emerges tangentially to the principal one.

In contrast to the rather simple determination of the critical load $\lambda_{\mathrm{c}}$ ( $\lambda_{\mathrm{c}}$ is given by $\left.\delta^{2} F\left(\lambda_{c}, v\right)=0\right)$ the determination of the coefficients in the postbifurcation expansion is a very cumbersome task. The analysis presented in this section is valid under the assumption that the incremental moduli tensor $\mathbf{L}$ depends smoothly on its arguments. Unfortunately, in the case of the simplest plasticity theories, the $\dot{\mathbf{F}}$ dependence of $\mathbf{L}$ is singular; for the simple one-dimensional case see (2.1). The corresponding generalization of the incremental moduli tensor $\mathbf{L}$ for a three-dimensional elastic-plastic solid with a smooth yield surface $\phi$ is $\mathbf{L}=\mathbf{L}^{\mathrm{e}}$ for $\phi<0$, and $\left.\mathbf{L}=\mathbf{L}^{\mathrm{e}}+H[\partial \phi / \partial \mathbf{T}): \dot{\mathbf{F}}\right\rceil\left(\mathbf{L}^{\mathrm{p}}-\mathbf{L}^{\mathrm{e}}\right)$ otherwise, where $\mathbf{L}^{\mathrm{e}}$ and $\mathbf{L}^{\mathrm{p}}$ are the elastic and plastic branches of the incremental moduli tensor. The presence of elastic reloading will complicate the analysis even further, as secn from the simple one-dimensional example presented in the previous section. The aforementioned complications are hardly surprising since, even for the simpler case of the plastic bifurcation of a material with a smooth yield surface, from a principal solution that does satisfy the total loading criterion everywhere, the computation of the coefficients of all (but the first) terms in the postbifurcation expansion involves laborious algebra (see for example, HuTCHInson (1973)). Moreover, the range of validity of the resulting asymptotic expansion is not known. In certain physically interesting cases which will be referred to subsequently, the range of validity of any postbifurcation expansion seems so limited as to make the whole postbifurcation analysis a futile mathematical exercise. 
Finally, a comment is made on an interesting subcase of the corner theory of ChristofFERSEN and Hutchinson (1979), for which the general bifurcation and postbifurcation analysis presented in this section can be carried out without any of the algebraic difficulties mentioned before. Recall that, for the aforementioned model, the rate potential $W$ can be put into the form

$$
W=\frac{1}{2} \dot{\mathbf{F}}:\left[\mathbf{L}^{\mathrm{e}}+f(\theta) \mathbf{C}\right]: \dot{\mathbf{F}},
$$

where $\mathbf{L}^{\mathrm{e}}(\mathbf{T}, \mathbf{F})$ is the instantaneous elastic moduli tensor, $\mathbf{C}\left(\mathbf{T}, \mathbf{F},{ }_{i} q\right)$ is the plastic moduli tensor for total loading and $f(\theta)$ is a smooth monotonically decreasing function of $\theta(0 \leqslant \theta \leqslant \pi)$ with $f(0)=1, f(\pi)=0$, where $\theta$ is a measure of deviation from proportional loading with $\theta$ a smooth function of $\mathbf{T}, \mathbf{F}, \mathbf{T}$. If $f(\theta)$ is taken to be a strictly monotonic and adequately smooth function of $\theta$, then the yield surface ceases to exist, and thus the dependence of the incremental moduli $\mathbf{L}$ as well as the internal variable ${ }_{i} q$ on their arguments is adequately smooth. Also, in this particular case and regardless of the prebifurcation solution (we may even have proportional loading), a smooth type bifurcation will be admitted by the equations. One such example can be found in ChristofFersen and HutCHINSON's (1979) analysis of the F.L.D. curve based on their corner theory. Although not explicitly mentioned in that reference, it has been observed (HUTCIInSON, 1981) that, in the particular case of the absence of a total loading conc in the corner theory, the bifurcation branch of the solution is tangent to the principal branch in a $\lambda-\|\overline{\mathbf{u}}\|$ diagram.

Quite recently, TVERGAARD (1982) investigated the nonaxisymmetric bifurcations of a compressed circular cylindrical shell from an axisymmetric prebifurcation configuration using the CHRISTOFFERSEN and HUTCHINSON (1979) corner theory. In most of these calculations the total loading cone was absent (no yield surface). Moreover, in view of the presence of axisymmetric imperfections in the undeformed state of the cylinder, there are significant deviations from proportional loading in the prebifurcation solution (the deviation from proportional loading increases with increasing values of the axisymmetric imperfection).

It was found that the smaller the imperfections, the bigger the difference between the $J_{2}$ deformation and $J_{2}$ corner theory predictions. In all the calculations presented the bifurcated branch of the solution appears to emerge tangentially from the principal branch. Especially for large imperfections (significant deviations from proportional loading prior to bifurcation) there is a clear indication of a cuspidal type bifurcation (see Fig. 2) in view of the slow separation (as the load increases) between the bifurcation and postbifurcation paths in a load-displacement diagram. For small axisymmetric imperfections, the bifurcation branch, although emerging tangentially, turns very rapidly away from the principal branch. It is this particular case, that justifies our earlier remark about the caution in seeking postbifurcation expressions for these more complicated plasticity theories.

\section{Conclusions}

In this work we have analyzed the bifurcation and postbifurcation of elastic--plastic solids whose behavior near the critical point could not be idealized as hypoelastic (in 
view of the non-trivial dependence of the prebifurcation solution and/or the incremental moduli on the strain rates).

The mathematical dependence of $\mathbf{L}$ on $\dot{\mathbf{F}}$ dictates the type of the perturbation expansion along the bifurcated branch of the solution whose main difference from Hill's theory is that both $\|\overline{\mathbf{u}}\|$ and $\|\dot{\overline{\mathbf{u}}}\|$ tend to zero as the time-like parameter $\lambda$ tends to its critical value $\lambda_{\mathrm{c}}$. For classical plasticity theories with smooth yield surface this type of behavior is encountered when unloading is present in the principal branch of the solution. In this case, using a simple example, we have shown the different possibilities existing for the postbifurcated solution (elastic reloading may or may not present, one or two symmetric bifurcated paths arise). We have also shown the applicability of our theory to the most recently developed phenomenological corner theories.

The dependence of the constitutive behavior of metals on the strain rate (of different type, of course, than the one adopted in (3.1)) can explain a number of stability related phenomena such as necking retardation in stretched bars and sheets (see HUTCHINSON and NEAL, 1977) or the non-observability of corners in yield surface experiments (see PAN and RICE, 1983). As a next step to explore further the effects of rate dependence on the stability behavior of metals, it will be interesting to investigate some more realistic constitutive equations using bifurcation analyses of the type presented in this work.

\section{ACKNOWLEDGMENTS}

Support for this work by the National Science Foundation under Contract NSF-MEA 8116449 is gratefully acknowledged. The author also acknowledges helpful discussions with Professor J. W. Hutchinson, Division of Applied Sciences, Harvard University.

\section{REFERENCES}

Christoffersen, $\mathbf{J}$.

Christoffersen, $\mathbf{J}$. and HuTCHINSON, J.

HiLL, R.

HILL, R.

HILL, R.

HUTCHINSON, J.

HutChinson, J.

Hutchinson, J.

HUTChINSON, J. and NEAL, $K$.

PAN, J. and RICE, J.

SHANLEY, F.

TRIANTAFYLLIDIS, N.

TRIANTAFYLLIDIS, N., NeEDleman, $A$. and

TVERGAARD, $V$.

TVERGAARD, $\mathrm{V}$.
1978 DCAMM Report No.147, Tech. Univ. of Denmark.

1979 J. Mech. Phys. Solids 27, 465.

$1956 J$ Jech. Phys. Solids 4, 247.

1958 J. Mech. Phys. Solids 6, 236.

1961 Muskhelisholli Anniv. Volume, S.I.A.M., Philadelphia, PA.

1973 J. Mech. Phys. Solids 21, 163.

1974 Adv. Appl. Mech. 14, 67.

1981 Private communication.

1977 Acta Metallurgica 25, 839.

1983 Int. J. Solids Struct., to appear.

1947 J. Aeronaut. Sci. 14, 261.

1980 J. Mech. Phys. Solids 28, 221.

1982 Int. I. Solids Struct. 18, p. 121.

1982 DCAMM Report No. 250, Oct. 1982. 